\title{
Design and analysis of a novel stator-doubly-fed doubly salient motor for electric vehicles
}

\author{
Ming Cheng ${ }^{\text {a) }}$ \\ Department of Electrical Engineering, Southeast University, Nanjing 210096, China \\ Ying Fan and K. T. Chau \\ Department of Electrical and Electronic Engineering, The University of Hong Kong, Hong Kong, China
}

(Presented on 11 November 2004; published online 17 May 2005)

\begin{abstract}
The design of a stator-doubly-fed doubly salient (SDFDS) machine is presented. The corresponding output power equation is analytically derived. The initial calculation of motor dimensions and parameters are also discussed. Moreover, finite element analysis of this motor is carried out, in which the interaction between the field flux and armature current flux as well as the magnetic saturation are taken into account. Hence, the characteristics of the proposed motor are deduced. A 12/8-pole SDFDS motor is designed and built for exemplification. Experimental results of the prototype are given to verify the theoretical analysis. (C) 2005 American Institute of Physics. [DOI: $10.1063 / 1.1853731]$
\end{abstract}

With ever increasing concerns of environmental protection and energy conservation, the electric vehicle (EV) has taken on an accelerated pace. To make the EV capable of competing with gasoline vehicles, the motor drive for the EV should be of high efficiency, high power density, high controllability, wide speed range, and maintenance-free. In order to pursue these goals, the permanent-magnet brushless (PMBL) motor drive and the switched reluctance (SR) motor drive have received much attention in the past decades. Moreover, the doubly-salient permanent magnet (DSPM) motor drive has recently been proposed which incorporates the advantages of both the PMBL and SR motor drives. ${ }^{1,2}$ Nevertheless, the DSPM motor drive still suffers from the drawbacks of uncontrollable PM flux and high PM material cost.

Having valuable research experience in the DSPM motor, we have proposed a machine topology, the so-called stator-doubly-fed doubly-salient (SDFDS) motor, ${ }^{3}$ which not only solves the fundamental problems of the DSPM machine, but also offers the possibility of optimizing the efficiency on-line. In Ref. 4, the basic operation principle of the SDFDS motor was introduced and a nonlinear magnetic circuit modeling method for the SDFDS motor was developed.

The main objective of this paper is first to present the design details of the SDFDS motor, thus providing the designer a practical way to make initial calculation of motor dimensions and parameters. A 12/8-pole SDFDS motor will be used for exemplification. Then, finite element analysis of this motor will be carried out, in which the magnetic saturation and the coupling between field current flux and armature current flux are taken into account. Finally, the experimental results on a prototype machine will be given to verify the theoretical prediction.

Figure 1 shows the schematic of a 12/8-pole (12 stator poles and 8 rotor poles) SDFDS motor. It adopts the same structure as a SR motor, namely saliency on both stator and

\footnotetext{
${ }^{a)}$ Author to whom correspondence should be addressed; Fax: 86-2583791696; electronic mail: mcheng@ seu.edu.cn
}

rotor. There are no windings or PMs on the rotor. Hence, the motor drive offers the capability of working at very high speed. The motor consists of two types of stator windings-a polyphase armature winding and a dc field winding. Since the dc current flowing through the field winding can be independently controlled, this machine can readily control the field flux for flux weakening operation and optimize the efficiency on-line. By using the similar derivation with that for the DSPM motor in Ref. 1, the sizing equation of the SDFDS motor can be deduced as

$$
D_{\mathrm{si}}^{2} l_{e}=\frac{P_{2}}{\frac{0.87 \pi^{2}}{120} \frac{p_{r}}{p_{s}} k_{d} k_{e} k_{i} A_{s} B_{\delta} n_{s} \eta},
$$

where $D_{\mathrm{si}}$ is the inner diameter of stator, $l_{e}$ the stack length, $P_{2}$ the output power, $p_{r}$ and $p_{s}$ the rotor and stator pole numbers, respectively, $A_{s}$ the electric loading of the armature winding of the stator, $B_{\delta}$ the airgap flux density, $n_{s}$ the rated speed in rpm, $\eta$ the motor efficiency, $k_{d}$ the flux leakage factor, $k_{e}=U / E$ the ratio of voltage to back EMF, $k_{i}$ $=I_{m} / I_{\mathrm{rms}}$ the ratio of current magnitude to rms value of current.

There is a wide range of possible combinations of phase windings, stator and rotor pole numbers that can be chosen for a SDFDS motor design. In accordance with the basic operation principle of the SDFDS motor, the general rela-

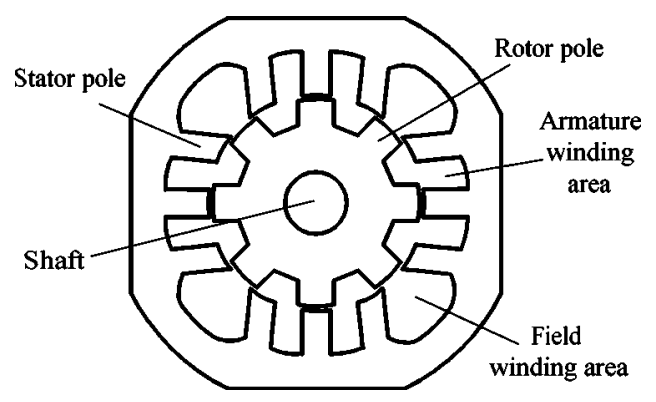

FIG. 1. Cross section of the 12/8-pole SDFDS motor. 
TABLE I. Technical data of the 12/8-pole SDFDS machine.

\begin{tabular}{lc}
\hline \hline & \\
Rated power (W) & 750 \\
Rated speed (r/min) & 1500 \\
Rated line-to-line peak voltage per phase (V) & 95 \\
Stator inner diameter (mm) & 75 \\
Stack length (mm) & 75 \\
Airgap length (mm) & 0.35 \\
Stator pole arc (deg) & 15 \\
Rotor pole arc (deg) & 20 \\
Stator pole height (mm) & 15 \\
Rotor pole height (mm) & 9 \\
Turn No. of armature winding/phase & 120 \\
Turn No. of field winding & 700 \\
\hline
\end{tabular}
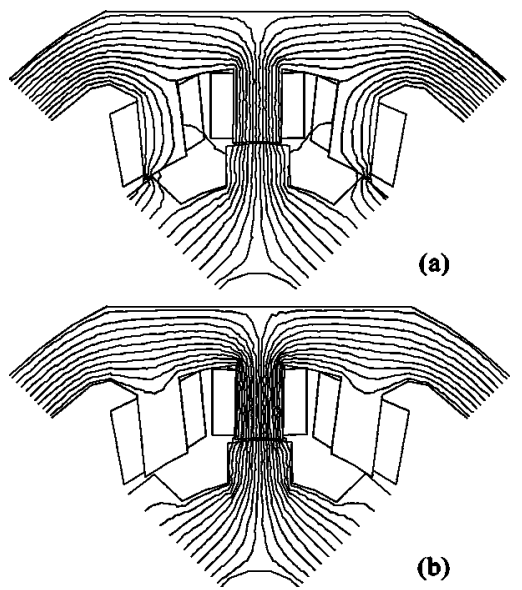

FIG. 2. (a) Field current flux. (b) Armature current flux of phase B.

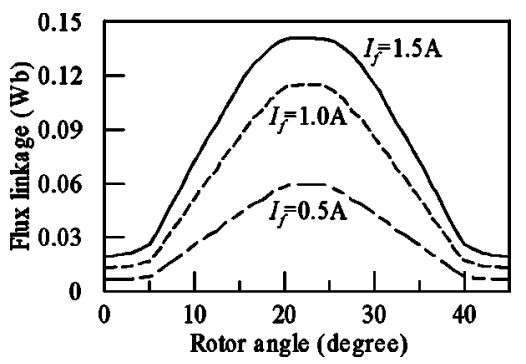

FIG. 3. Flux linkage versus rotor position angle.

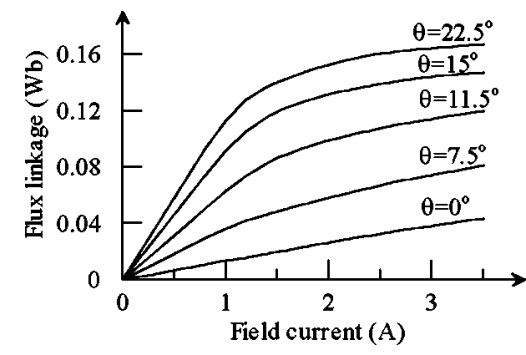

FIG. 4. Flux linkage versus field current.

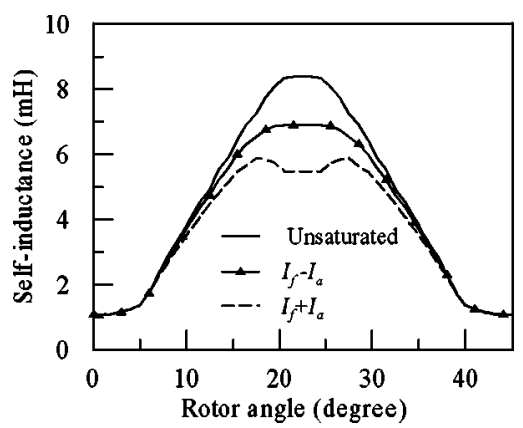

FIG. 5. Self-inductance characteristics $\left(I_{f}=1 \quad \mathrm{~A}, I_{a}=2 \mathrm{~A}\right)$.

tionships among stator pole number $p_{s}$, rotor pole number $p_{r}$, and phase number $m$ are given by

$$
\left\{\begin{array}{l}
p_{s}=2 m k \\
p_{r}=p_{s} \pm 2 k
\end{array}\right.
$$

where $k$ is a positive integer. When the motor runs at the speed of $n$, the commutating frequency of any phase is

$$
f_{\mathrm{ph}}=p_{r} n / 60 .
$$

To minimize the switching frequency and hence the iron losses in poles and yokes as well as the loss in power switches, the number of rotor poles should be selected as small as possible. Therefore, the number of rotor poles is usually less than that of stator poles. To make the motor capable of starting itself in either forward or reverse direction, the phase number should be equal to or greater than three. Thus, $p_{s} / p_{r}=6 / 4,8 / 6$, and $12 / 8$ are possible configurations of the SDFDS motor. As compared to the 3-phase 6/4-pole one, the 3-phase 12/8-pole SDFDS motor possesses shorter flux paths in yoke due to the fact that the circumference of stator is split into four segments by 4 field coils (only two in 6/4-pole machine), resulting in less magnetic potential drop and iron losses. Moreover, because the flux per magnetic pole is halved in 12/8-pole machine, the width of both stator yoke and teeth is almost one-half of those of a 6/4-pole machine. This allows greater inner stator diameter and hence greater rotor diameter. Therefore, higher torque density can be achieved. Furthermore, less width of stator teeth results in shorter end part of phase windings, leading to less copper consumption and resistance of windings. Hence, higher efficiency can be expected for the SDFDS machine.

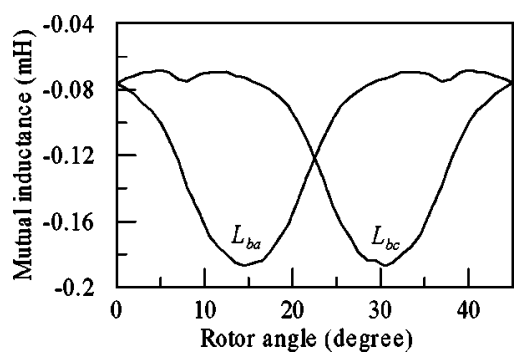

FIG. 6. Mutual inductance characteristics. 


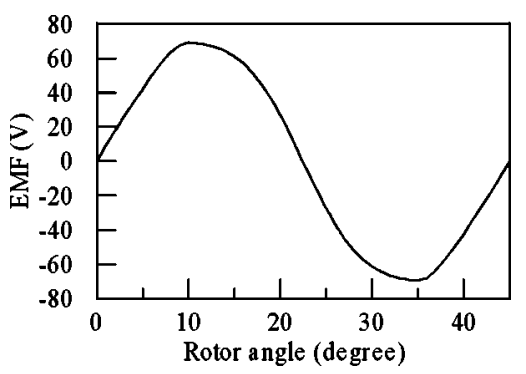

FIG. 7. Predicted back EMF waveform at $1500 \mathrm{r} / \mathrm{m}$ and $I_{f}=1 \mathrm{~A}$.

To minimize the permeance associated with the minimum field flux at unaligned rotor position, pole arcs should comply with the following condition:

$$
\beta_{s}+\beta_{r}<\theta_{\mathrm{cr}}=360^{\circ} / p_{r} .
$$

The turns of the armature winding per phase can be initially chosen by ${ }^{1}$

$$
w=\frac{0.8 U \theta_{w} \eta}{0.87 k_{d} \frac{c_{s} \pi D_{\mathrm{si}}}{p_{s}} l_{e} \frac{2 \pi n_{s}}{60}}
$$

The turns of the field winding may be designed in such a way that the ampere-turns of the field winding should achieve an airgap magnetic density roughly equal to $1.5 \mathrm{~T}$ under the stator teeth.

In order to leave sufficient room for the field windings, three stator teeth in each magnetic pole region are purposely designed to be nearly parallel, as shown in Fig. 1.

Based on the above design procedure, the dimensions and parameters of a 12/8-pole SDFDS machine are obtained. The major design data are listed in Table I.

Two-dimensional (2D) finite element analysis (FEA) is adopted to determine the magnetic field distribution of the SDFDS motor. Due to the periodic configuration of the 12/8-pole machine, namely, four field poles and twelve armature poles, the region of interests for FEA is one-quarter of the whole motor cross section, which complies with the semiperiodic boundary condition.

The magnetic field distributions of the SDFDS motor at different load conditions and rotor position angles are analyzed. Figure 2 shows the distributions at the instant that a rotor pole aligns with the stator pole of phase $\mathrm{B}$, in which Fig. 2(a) is the field produced by field current at no-load, whereas Fig. 2(b) is the field produced by armature current of phase B only.

Based on the results of FEA, the characteristics of the SDFDS machine, including field flux linkage, self-

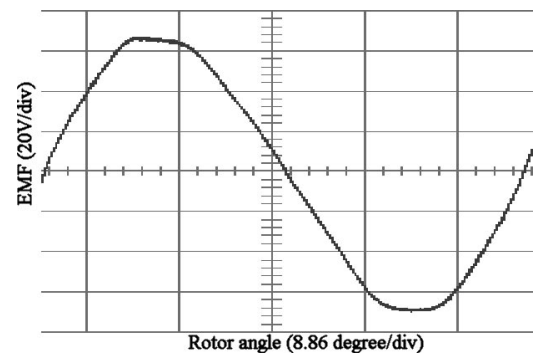

FIG. 8. Measured back EMF at $1500 \mathrm{r} / \mathrm{m}$ and $I_{f}=1 \mathrm{~A}$. inductance, mutual inductance, back EMF, can be deduced. Figure 3 shows the flux linkage versus rotor position angle at different field currents, while Fig. 4 gives the flux linkage versus field current at different rotor position angles, where the rotor position angle is defined as the angle between the stator pole center line and the rotor slot center line. It can be seen that the magnetic saturation occurs for the cases of large rotor position angles, namely the stator pole overlapping with rotor pole, when the field current increases.

Figure 5 illustrates the self-inductance of the armature winding, where the unsaturated characteristic is obtained at field current of $1 \mathrm{~A}$. To calculate the saturated inductance, the cross coupling between the field flux and armature flux is taken into account. In Fig. 5, the saturated inductances at field current of $1 \mathrm{~A}$ and armature current of $2 \mathrm{~A}$ are also shown, where " $I_{f}+I_{a}$ " and " $I_{f}-I_{a}$ " denote the strengthening and weakening actions of the armature flux to the field current flux, respectively. It is seen that the saturated inductance of the SDFDS motor is a function of not only rotor position, but also the strengthening and weakening actions of the armature current flux to the field current flux. Moreover, Fig. 6 shows the mutual inductance between armature phases, indicating that the mutual inductance in the proposed SDFDS machine is negligible as compared to the self-inductance.

According to the flux linkage characteristics in Fig. 3, the back EMF can readily be deduced by

$$
e=d \psi_{f} / d t=\left(d \psi_{f} / d \theta\right) \omega_{r}
$$

Figure 7 shows the predicted EMF waveforms of the machine with a rotor skewed by 10 mechanical degrees.

To verify the theoretical analysis and design, a 12/8-pole SDFDS motor with a rotor skewed by $10^{\circ}$ was built and tested. The measured back EMF waveform is shown in Fig. 8 , verifying the theoretical analysis.

In this paper, the design and analysis of a SDFDS motor has been presented. The sizing equation and the relationships between the main design parameters and performance requirements are established, providing a practical way for the designer to make initial calculations of the motor frame and winding turns. A 12/8-pole SDFDS motor has been designed and built for evaluation. Moreover, the finite element analysis of the proposed motor has been carried out. Hence, the static characteristics of the motor have been deduced, showing that the inductance of the SDFDS motor depends on both the rotor position and current, and that the mutual inductance is negligible. The experimental results have verified the theoretical analysis.

This work was supported in part by grants from NSFC, China (Project No. 50377004, 50337030) and by a grant from RGC of Hong Kong (Project No. HKU 7035/01E).

${ }^{1}$ Ming Cheng, K. T. Chau, and C. C. Chan, IEEE Trans. Magn. 37, 3012 (2001).

${ }^{2}$ Ming Cheng, K. T. Chau, C. C. Chan, and Qiang Sun, IEEE Trans. Ind. Appl. 39, 1363 (2003).

${ }^{3}$ Ming Cheng and E. Zhou, Chinese Patent No. ZL99114598.4, May 2003.

${ }^{4}$ K. T. Chau, Ming Cheng, and C. C. Chan, IEEE Trans. Magn. 38, 2382 (2002). 